\title{
A single-molecule multicolor electrochromic device generated through medium engineering
}

\author{
Yu-Mo Zhang ${ }^{1}$, Xiaojun Wang ${ }^{1}$, Weiran Zhang ${ }^{1}$, Wen $\mathrm{Li}^{1}$, Xiaofeng Fang ${ }^{1}$, Bing Yang ${ }^{1}$, Minjie $\mathrm{Li}^{1}$ and Sean \\ Xiao-An Zhang ${ }^{1,2}$
}

Multicolor organic electrochromic materials are important for the generation of full-color devices. However, achieving multiple colors using a single-molecule material has proved challenging. In this study, a multicolor electrochromic prototype device is generated by integrating medium engineering/in situ 'electro base'/laminated electrode technologies with the simple flying fish-shaped methyl ketone TM1. This multicolor electrochromic (green, blue and magenta) device is durable and has a high coloration efficiency $\left(350 \mathrm{~cm}^{2} \mathrm{c}^{-1}\right)$, a fast switching time $(50 \mathrm{~ms})$ and superior reversibility. This study is a successful attempt to integrate solvatochromism and basochromism in an electronic display. This integration not only introduces a new avenue for color tuning, in addition to the structural design of the colorant, but will also inspire further developments in the tuning of many other properties by this medium engineering approach, such as conductance and the redox property, and thereby accelerate versatile applications in data recording, ultrathin flexible displays, and optical communication and sensing.

Light: Science \& Applications (2015) 4, e249; doi:10.1038/Isa.2015.22; published online 13 February 2015

Keywords: electrochromic materials; laminated electrodes; microenvironment; multicolor devices; solvatochromic materials

\section{INTRODUCTION}

Owing to the increasing demand for low-power, ultrathin, flexible electronic displays, organic electrochromic materials ${ }^{1-3}$ have become a research focus because of their distinct merits: low weight, high contrast, wide viewing angle, flexibility and the potential for low power consumption. Because the realization of a multicolor switch is preferred in the majority of their applications in displays, ${ }^{4-10}$ various electrochromic materials including polymers, ${ }^{11,12}$ small organic molecules $^{13,14}$ and metal-organic complexes, ${ }^{15}$ and technologies aimed at multicolor switches have been intensively explored. Transition metals based on metal-organic complex multicolor electrochromic materials exhibit attractive properties, such as high stability and chemiluminescence. ${ }^{16}$ However, the expense and scarcity of the precious metals, such as ruthenium, ${ }^{15,16}$ osmium ${ }^{17}$ and iridium, ${ }^{18}$ limit their practical applications. Polymeric multicolor electrochromic materials with different electrochromic activation units ${ }^{19-21}$ possess the advantages of easy processing, diverse resources and facile color tunability. However, some intrinsic problems, such as the lack of colorless states, poor transparency, poor color purity and complicated synthesis procedures, hinder their applications. Compared with electrochromic polymers, small organic color switches possess the advantages of low cost, good color purity, distinct transition from colorless to colored states and fine tunability of their photoelectronic properties via easy structure modifications. The lack of multicolor abilities is their intrinsic problem; only a few organic molecules ${ }^{22,23}$ can exhibit limited color tunability within a single device, and the resulting colors remain deficient regarding the three primary colors for display.

To address these challenges, we discuss a new strategy to achieve multicolor properties from a single small molecule. The multicolor electrochromic prototype device is realized by the methyl ketone TM1 (Figure 1). Variable colors were achieved by tuning the ratios of two plasticizers (propylene carbonate (PC)/diglycol) in a polymethyl methacrylate (PMMA) matrix in different layers of a multi-electrode device. Our multi-electrode approach is conceptually different from the traditional multi-electrode technology for creating variable colors with various molecules. ${ }^{24,25}$ We can generate multiple color from the same molecule by simply tuning its microenvironment. With this method, we were able to achieve variable colors, including magenta, green and blue, with a fast switching time $(50 \mathrm{~ms})$, high coloration efficiency and good reversibility. To our knowledge, this is first example of high-quality multicolor switching with a simple molecule by this new multi-electrode method.

\section{MATERIALS AND METHODS}

\section{Synthesis and characterization}

The synthetic route that was employed to prepare the compound TM1 is shown in Supplementary Information. TM1 was obtained from 1(9,9-dihexyl-7-iodo-9H-fluoren-2-yl)-2-(2-nitrophenyl)ethanone (M1)

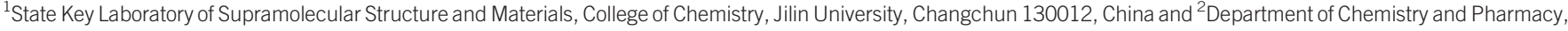
Zhuhai College of Jilin University, Zhuhai 519041, China

Correspondence: M Li, State Key Laboratory of Supramolecular Structure and Materials, College of Chemistry, Jilin University, Changchun 130012, China

E-mail: liminjie@jlu.edu.cn

SXA Zhang, State Key Laboratory of Supramolecular Structure and Materials, College of Chemistry, Jilin University, Changchun 130012, China

E-mail: seanzhang@jlu.edu.cn

Received 7 August 2014; revised 26 November 2014; accepted 5 December 2014; accepted article preview online 11 December 2014 


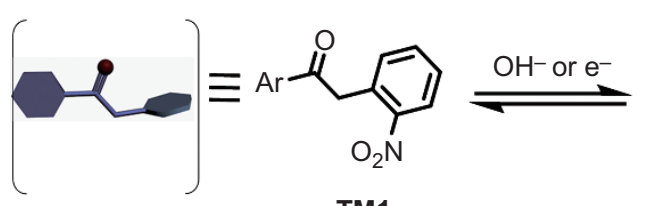

TM1<smiles>CC(=O)[Te]</smiles>

TM2<smiles>O=[N+]([O-])c1ccccc1/C=C/c1ccccc1</smiles>

TM3

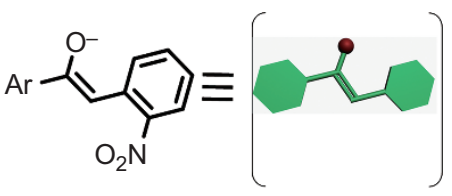

TM1-enolate

Figure 1 The structures of TM1, TM1-enolate, TM2 and TM3 (the red spheres represent oxygen atoms).

and N,N-dibenzyl-4-((trimethylsilyl)ethynyl)aniline (M2) with a high yield $(95 \%)$ by a modified one-pot process of Sonogashira coupling. We combined deprotection of the trimethylsilyl-protecting group and the subsequent coupling in one step to simplify the process and improve the yield. The compounds TM2, TM3 and other reference molecules (Figures 1 and 2) were synthesized by some general methods, as previously described. ${ }^{26,27}$ All compounds were purified and completely characterized by ${ }^{1} \mathrm{H}-\mathrm{NMR},{ }^{13} \mathrm{C}-\mathrm{NMR}$ and mass spectrometry. We refer to TM1 as the 'flying fish molecule' due to the interesting appearance of its crystal structure, as depicted in Figure 2b and Supplementary Fig. S1.

Preparation of sandwich-type indium tin oxide (ITO) cells A mixture of PMMA (0.5 g, 30\%, wt-\%), PC/diglycol (1 mL, 70\%, wt-\%, different PC/diglycol fractions), $\mathrm{TBAPF}_{6}(0.1 \mathrm{M})$ and TM1 $\left(1.0 \times 10^{-2} \mathrm{M}\right)$ in $10 \mathrm{~mL}$ acetonitrile was stirred for $24 \mathrm{~h}$. The electrochromic solution was dropcast onto ITO glass at $45{ }^{\circ} \mathrm{C}$ for $2 \mathrm{~h}$. The thickness was set to approximately $0.1 \mathrm{~mm}$. For a monolayer device, the electrochromic film was laminated between two ITO electrodes. For a multi-layered electrochromic device, the electrochromic films $(0.1 \mathrm{~mm})$ were laminated between every two ITO electrodes.

\section{Spectroelectrochemical characterization}

Changes in absorption during the cyclic voltammogram were measured in situ using a home-made spectroelectrochemical cell and a spectrophotometer. $^{26,28}$ The multi-potential modes of a bio-logic electrochemical work combined spectrophotometer were employed to measure the absorption spectra and lifetime of the sandwich-type ITO cells in situ.
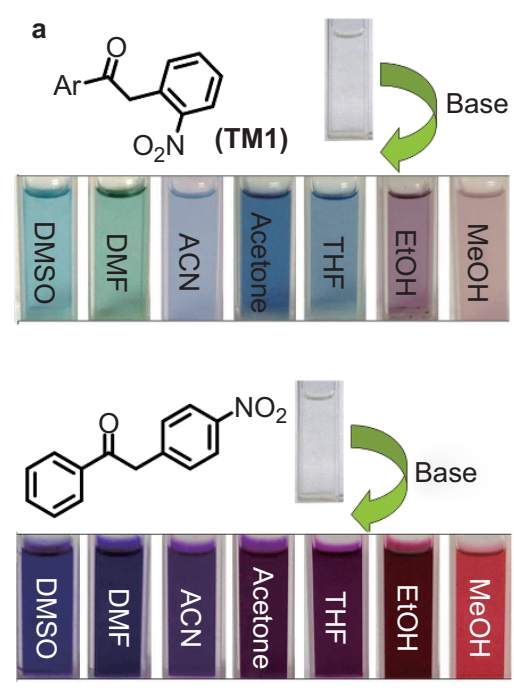<smiles>O=C(Cc1ccc(C(F)(F)F)cc1)c1ccccc1</smiles>
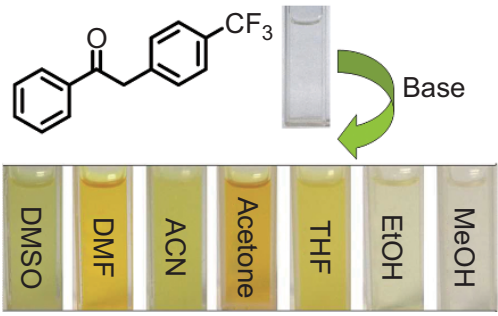
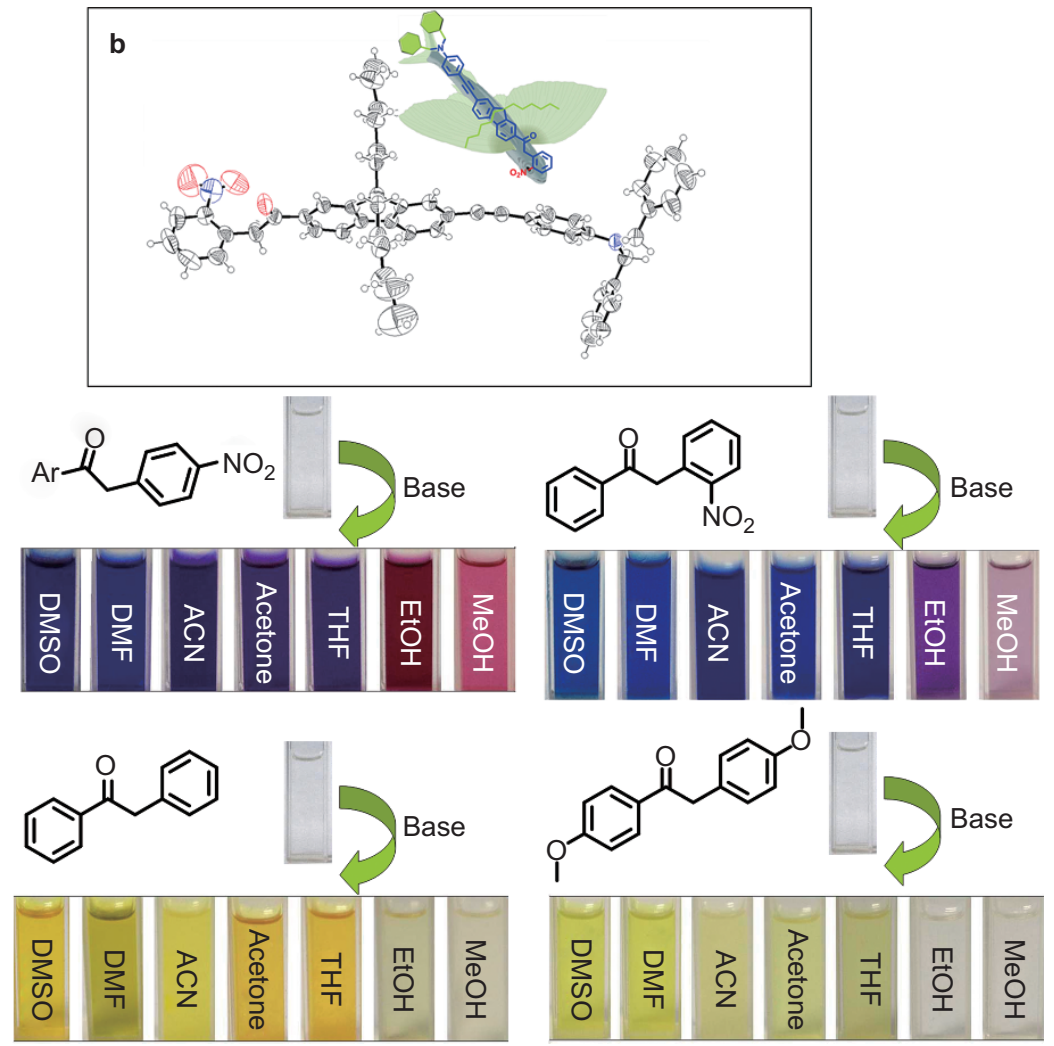

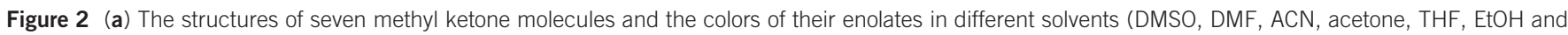

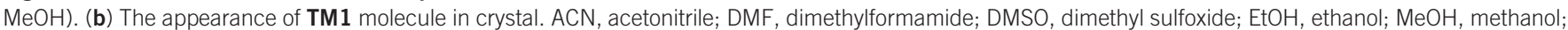
THF, tetrahydrofuran. 


\section{RESULTS AND DISCUSSION}

Design and synthesis of the switchable molecule

The method demonstrated in this paper originates from an inspiration derived from solvatochromism. The absorption and emission spectra of some solvatochromic materials ${ }^{29,30}$ are highly dependent on the properties of their solvents, such as polarity, hydrogen bonding and other intermolecular forces; these qualities inspired us to consider whether a multicolor electronic display could be constructed from a single color switch based on the principle of solvatochromism. The success of this attempt will provide a new approach to achieving multiple colors, and will stimulate and accelerate further development of versatile electrochromic materials. However, several barriers prevent the implementation of this idea: (i) most electrochromic organic materials do not possess the required prominent solvatochromic properties; (ii) most solvatochromic dyes do not possess electrochromic properties. And In addition they usually contain intrinsic color, which obviously do not meet the requirement of transparent state in the display; Leuco dyes satisfy the transparent requirement, but are usually not electrochromic; and (iii) the creation of different microenvironments of 'solid solvents' in a single cell is currently challenging. We envision that the first two problems could be solved by the reversible 'electrobase/acid' that we explored in situ with some $\mathrm{pH}$-sensitive leuco dyes; ${ }^{26,28}$ the last problem can be technically overcome using laminated cells. ${ }^{24,25}$

Based on our previous study, ${ }^{26,27}$ we discovered that some methyl ketone molecules exhibit basochromic properties, which can be easily switched by electrochemically generated base/acid (electrobase/acid) and show a distinct color change, acceptable stability and reversibility. A significant change in molecular polarity was also observed during the switching. These changes prompted us to utilize them to achieve the solvatochromic multicolor objective. For proof-of-principle, a series of methyl ketone molecules were designed and synthesized to verify our assumption and prove the reaction mechanism. The results of the solution test show that all the synthesized methyl ketone molecules had a significant effect on the basochromism and solvatochromic properties (Figure 2), and that TM1, which has a 2-nitro group and large conjugate backbone, is a favorable candidate for further investigation of a multicolor device, because of its significant color variation in different solvents. For example, it shows green in DMSO (dimethyl sulfoxide) or DMF (dimethylformamide), blue in $\mathrm{CH}_{3} \mathrm{CN}$, acetone or THF (tetrahydrofuran) and magenta in ethanol or methanol. Therefore, the following investigation of multicolor electrochromic devices is based on TM1.

\section{Solution evaluation of the molecular color switching}

Because basochromism, electrochromism and solvatochromism of the molecules are the three essential properties required to fulfill our goal, these properties of the TM1 were examined in solution first. Considering DMF as an example of a solvent, the colorless TM1 exhibits a very pleasant green $(617 \mathrm{~nm})$ color after the addition of base (Figure 3a), which indicates that the methyl ketone (TM1)-enolation (TM1-enolate, Figure 1a) tautomerization has been induced by the
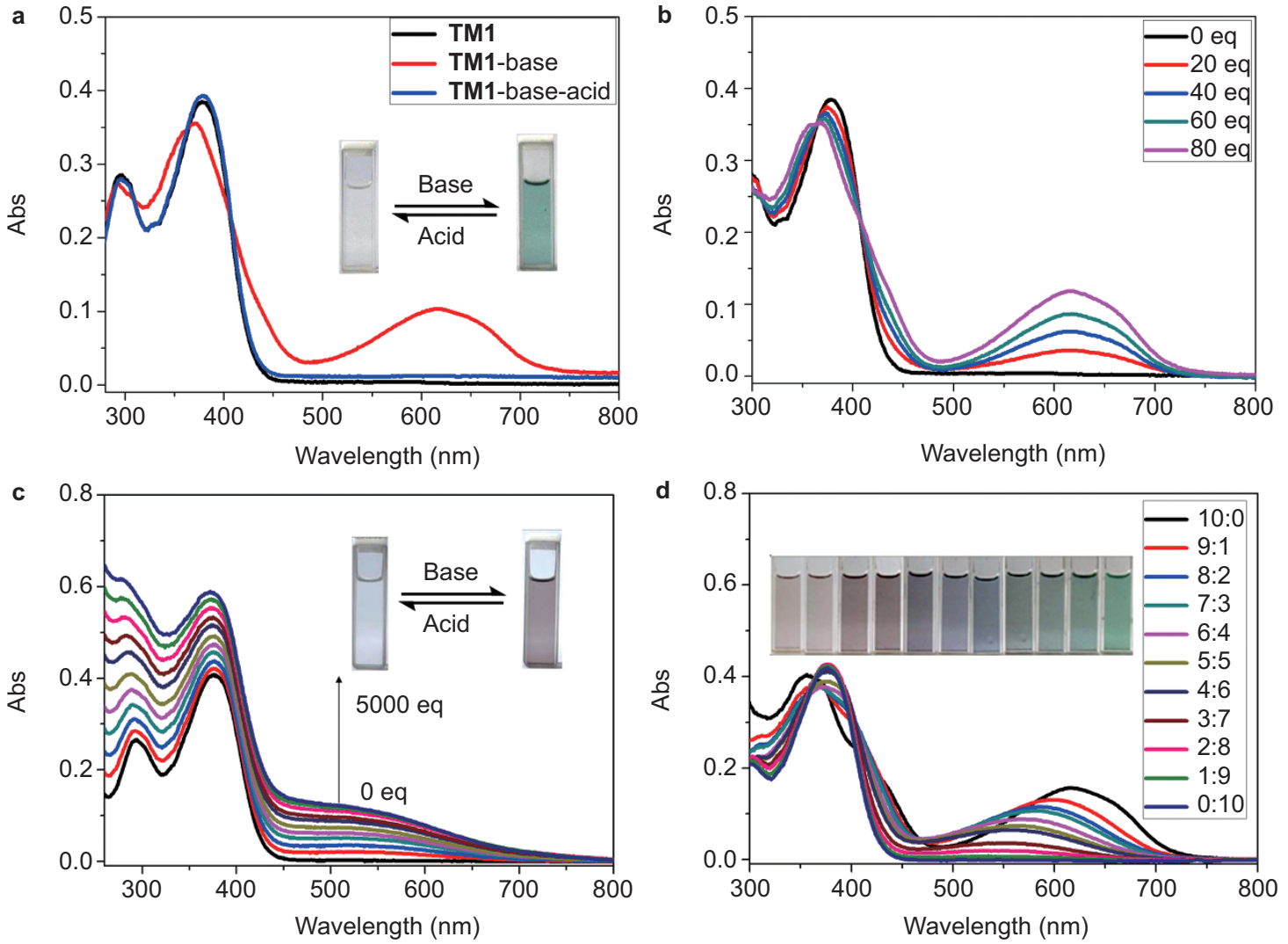

Figure 3 (a) Absorption spectra of TM1 $\left(1.0 \times 10^{-5} \mathrm{~mol} \mathrm{~L}^{-1}\right.$, black), base (potassium tert-butoxide, red) added and then treated with $\mathrm{CH}_{3} \mathrm{COOH}$ (blue) in DMF. (b) Absorption spectra of TM1 $\left(1.0 \times 10^{-5} \mathrm{~mol} \mathrm{~L}^{-1}\right)$ in the presence of potassium tert-butoxide ( 0 eq, 20 eq, 40 eq, 60 eq and 80 eq) in DMF. (c) Absorption spectra of TM1 $\left(1.0 \times 10^{-5} \mathrm{~mol} \mathrm{~L}^{-1}\right)$ treated with different equivalent potassium tert-butoxide (500 eq interval) in ethanol. (d) Absorption spectra of TM1 (1.0 $10^{-5}$ mol $\mathrm{L}^{-1}$ ) treated with 80 eq potassium tert-butoxide in DMF/ethanol mixtures with different ethanol fractions. DMF, dimethylformamide. 
addition of base. The tautomerization equilibrium is dependent on the concentration of base (Figure $3 \mathrm{~b}$ ), and the color can be easily reverted by the addition of acid. If the solvent is changed to protic ethanol, ketone-enolation tautomerization can also be triggered; however, more base is required and a different absorption band appears without a distinct peak, which results in a magenta color (Figure 3c). Therefore, we show that both the basochromic and solvatochromic behaviors can be achieved within the same molecule of TM1 with an extensive range of colors.

To completely understand the basochromic and solvatochromic behaviors of TM1, theoretical simulation with density functional theory calculations provides additional insight into the correlation between the absorption bands and their structures. ${ }^{31}$ As shown in Supplementary Table S1, HOMO (highest occupied molecular orbital) for TM1-enolate is primarily localized on its oxygen anion portion, and LUMO (lowest unoccupied molecular orbital) is primarily localized on the nitro group. Thus, the absorption band for TM1enolate at $617 \mathrm{~nm}$ is assigned to the intramolecular charge transfer bands from the oxygen anion of enolate moiety to nitrobenzene moiety. As shown in Supplementary Table S2, the maximum absorption wavelength of TM1-enolate decreases from $618 \mathrm{~nm}$ (green) to $575 \mathrm{~nm}$ (blue) when the solvent polarity decreases from 7.2 (DMSO) to 2.4 (toluene) in aprotic solvents, which is an important property for intramolecular charge transfer. ${ }^{32,33}$ However, some exceptions to this trend are noted. For example, the TM1-enolate shows a maximum absorption at $603 \mathrm{~nm}$ in dichloromethane which exhibits low polarity. This finding indicates that the solvent polarity is not the only determinant factor; other unknown factors may serve a role.

Regarding the effect of hydrogen bonding on the maximum absorption wavelength of TM1-enolate, the ethanol molecules were considered in the calculation to provide hydrogen bonds with TM1-enolate. As shown in Supplementary Table S3, the larger is the number of ethanol molecules that were considered, the larger is the HOMOLUMO gap. This trend is consistent with another calculated result-that the contribution from the oxygen anion to the HOMO decreases when one or more ethanol molecules are considered. This contribution inevitably decreases the intramolecular charge transfer from the moiety of its oxygen anion to the moiety of its nitrobenzene after more hydrogen bonds are formed and consequently produces an increase in the HOMO-LUMO gap. This finding explains the observed blue-shift in the absorption of TM1-enolate in ethanol compared with in DMF owing to the anticipated hydrogen-bonding interactions. In addition, the $\mathrm{C}-\mathrm{O}$ bond length of the oxygen anion increases from $1.276 \AA$ to $1.310 \AA$ when more hydrogen bonds are formed with the TM1-enolate (Supplementary Table S4). This result provides undisputable evidence of the reduction in the intramolecular charge transfer. The calculation results further show that the larger is the number of ethanol molecules, the larger are the interaction energies (from $-6.83 \mathrm{kcal} \mathrm{mol}^{-1}$ to $-17.84 \mathrm{kcal} \mathrm{mol}^{-1}$ ) of TM1-enolate and ethanol. This finding indicates that the hydrogen bonding interaction serves a crucial role in the blue shifting of the maximum absorption wavelength of TM1-enolate in protic solvents.

These results indicate that the effect of hydrogen bond and solvent polarity serve a crucial role in the color absorption of TM1-enolate, which prompted us to explore the possibility of obtaining multiple colors by manipulating both effects in a bi-solvent system. Further exploration with TM1-enolate in a series mixture of DMF and ethanol confirmed our conjecture that it should be possible to obtain various colors by the simple tuning of the composition of its solvents.
As shown in Figure 3d, the maximum absorption wavelength of TM1-enolate continuously decreases with a decrease in the DMF fraction in the bi-solvent system, and the color can be tuned from green $(617 \mathrm{~nm})$ to blue $(575 \mathrm{~nm})$ to magenta $(537 \mathrm{~nm})$. This breakthrough is exciting for a single-molecule multicolor device and will surely lead and accelerate future development of molecular color switches in displays.

The electrochromic property of TM1 is next studied by cyclic voltammetry (CV) in DMF. A green color that appears at $-1.0 \mathrm{~V}$ and disappears at $-0.25 \mathrm{~V}$ confirms that the color switch can be reversibly triggered by an electrical method (Figure 4). Electrochromism is discussed in the next section.

\section{Multicolor electrochromic investigation in a thin-film device}

The switchability of TM1 in a thin-film device is first examined. We initially fabricated the electrochromic thin-film devices in a media of PMMA/PC/TBAPF 6 sandwiched in an ITO cell. The thin-film device rapidly switches between a colorless state and a colored state. As depicted in Supplementary Fig. S2, the discernible color change of the thin-film device can be obtained by a $-5.0 \mathrm{~V}$ voltage for $50 \mathrm{~ms}$. The absorbance of the thin film at $617 \mathrm{~nm}$ exhibits no distinct degradation during 85 test cycles, as monitored by in situ absorption spectroscopy (Figure 5a). The encouraging results demonstrate that the electrochromic switching of TM1 in a thin-film device is not only feasible but also completely reversible.

The efficiency of an electrochromic device can be compared by the coloration efficiency, which is employed to describe the change in the number of color centers as a function of the charge. Although the
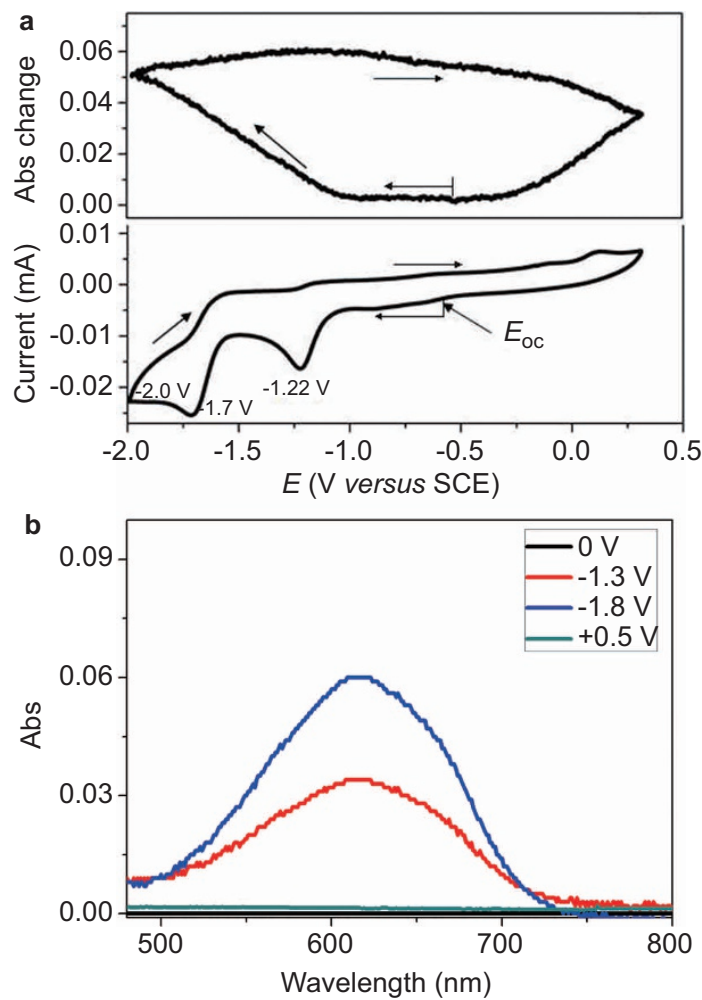

Figure 4 (a) Change in the absorption at $617 \mathrm{~nm}$ (top) and the cyclic voltammogram (bottom) of TM1 $\left(1.0 \times 10^{-3} \mathrm{~mol} \mathrm{~L}^{-1}\right)$ in DMF at a scan rate of $50 \mathrm{mV} \mathrm{s}^{-1}$. (b) Absorption spectra of TM1 in DMF at $0 \mathrm{~V}$ (black), $-1.3 \mathrm{~V}$ (red) and $-1.8 \mathrm{~V}$ (blue) at approximately $60 \mathrm{~s}$, then at $0.5 \mathrm{~V}$ (green). DMF, dimethylformamide; SCE, saturated calomel electrode. 

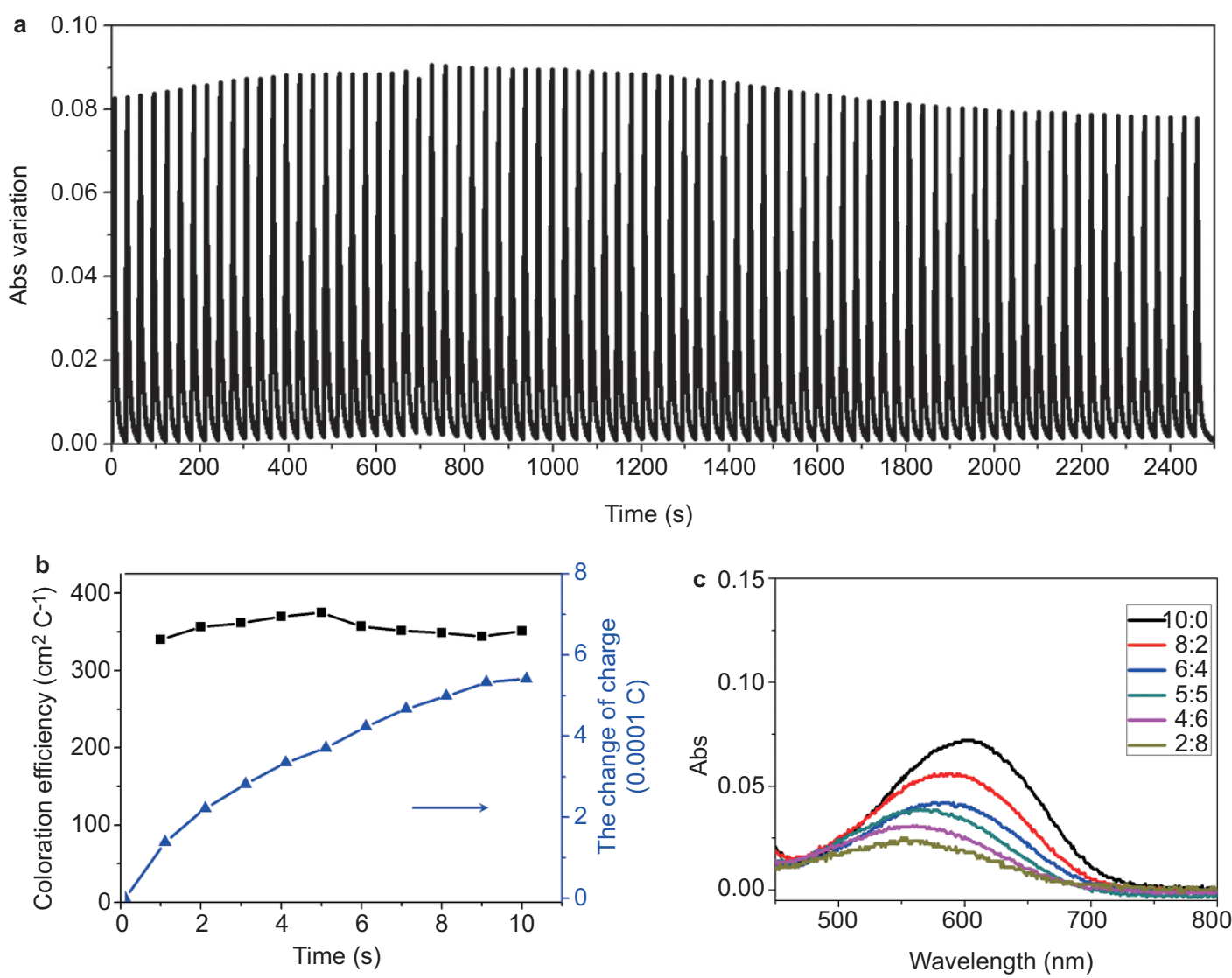

Figure 5 (a) Absorbance variation $(617 \mathrm{~nm})$ in the PMMA/PC/TBAPF 6 film of TM1 with the switch cycles generated by alternating $-3.0 \mathrm{~V}(5 \mathrm{~s})$ and $2.0 \mathrm{~V}(25 \mathrm{~s})$ voltages. (b) Coloration efficiency (black) and the charge change (blue) as a function of switch time for EC thin film. (c) Absorption spectra of TM1 stimulated by - $3.0 \mathrm{~V}$ voltage in PMMA film with different PC/diglycol fractions. EC, electrochromic; PC, propylene carbonate; PMMA, polymethyl methacrylate.

coloration efficiency has been defined in several ways, all definitions are suitable only for an electrochromic polymer. ${ }^{34}$ The most common definition for the coloration efficiency of an electrochromic polymer is the change in absorbance $(\Delta A)$ obtained for a specific amount of injected charge per unit area $(Q) .{ }^{35}$ This definition is obviously not suitable for small organic electrochromic materials because not all of injected charges are used for electroreactions in the presence of a large amount of electrolytes. To apply to the system of small organic electrochromic materials, we have modified the definition of the coloration efficiency of the device by eliminating the background interference of electrolytes. The newly defined coloration efficiency $\eta$ is given by the optical absorbance change $(\Delta A)$ obtained for the change in injected charges per unit area $(\Delta Q)$ only, where $\Delta Q$ is calculated by subtracting the injected charge per unit area $\left(Q_{2}\right)$ of a blank device with no switchable molecules from the injected charge per unit area $\left(Q_{1}\right)$ of electrochromic device subtracts.

$$
\eta=\Delta A / \Delta Q=\Delta A /\left(Q_{1}-Q_{2}\right)
$$

In this newly defined method, the $\eta$ for the TM1 device is approximately $350 \mathrm{~cm}^{2} \mathrm{C}^{-1}$, as shown in Figure $5 \mathrm{~b}$, and remains nearly constant during the entire extended switching test. This finding indicates not only the stable electrochromic performance of the TM1 device, but also that this modified definition for coloration efficiency is feasible to our system.

Currently, all test results from the PMMA thin-film device of TM1 are satisfactory. Previous parallel research by our group assures us that the microenvironment in solution can be imitated in thin film. ${ }^{36}$ These results encouraged us to explore whether we could freely tune the microenvironment effect of TM1 to produce variable colors in a thin-film device. To simulate the situation of ethanol in DMF, we used a mixture of PC and diglycol as additives for tuning the property of the medium. The thin-film devices performed exactly as we anticipated after extensive attempts to vary the percentage and ratio of the additives. As shown in Figure $5 c$, the maximum absorption wavelength of the ITO device continuously decreased with an increase in the diglycol fraction in its PMMA film, and the electrically switched color could be tuned from green to blue to magenta. These fascinating results prove the feasibility of constructing a single-molecule multicolor device.

To demonstrate its potential use in a display, we constructed a simple prototype ITO device based on the multi-layered and multielectrode strategy. For illustration purposes, the device structure is simplified using three electrochromic layers and four orthogonal thin-film electrodes, as shown in Figure 6a; two of the electrodes are single-sided conductive electrodes and the remaining two are doublesided conductive electrodes. The additives ratio of $\mathrm{PC}$ and diglycol is $10: 0,6: 4$ and $2: 8$ for the green, blue and magenta electrochromic layers, respectively. The initial state of the electrochromic device is colorless at $0 \mathrm{~V}$, as shown in Figure 6b. The device can be easily switched to achieve either one of three primary colors (blue, green and magenta) or secondary colors by activating one or more sets of its electrodes. To verify the stability and reversibility of the multi-layered electrochromic device, the absorbance $(583 \mathrm{~nm}$ blue, $617 \mathrm{~nm}$ green, $551 \mathrm{~nm}$ red) of each layer was monitored by ultraviolet-visible 

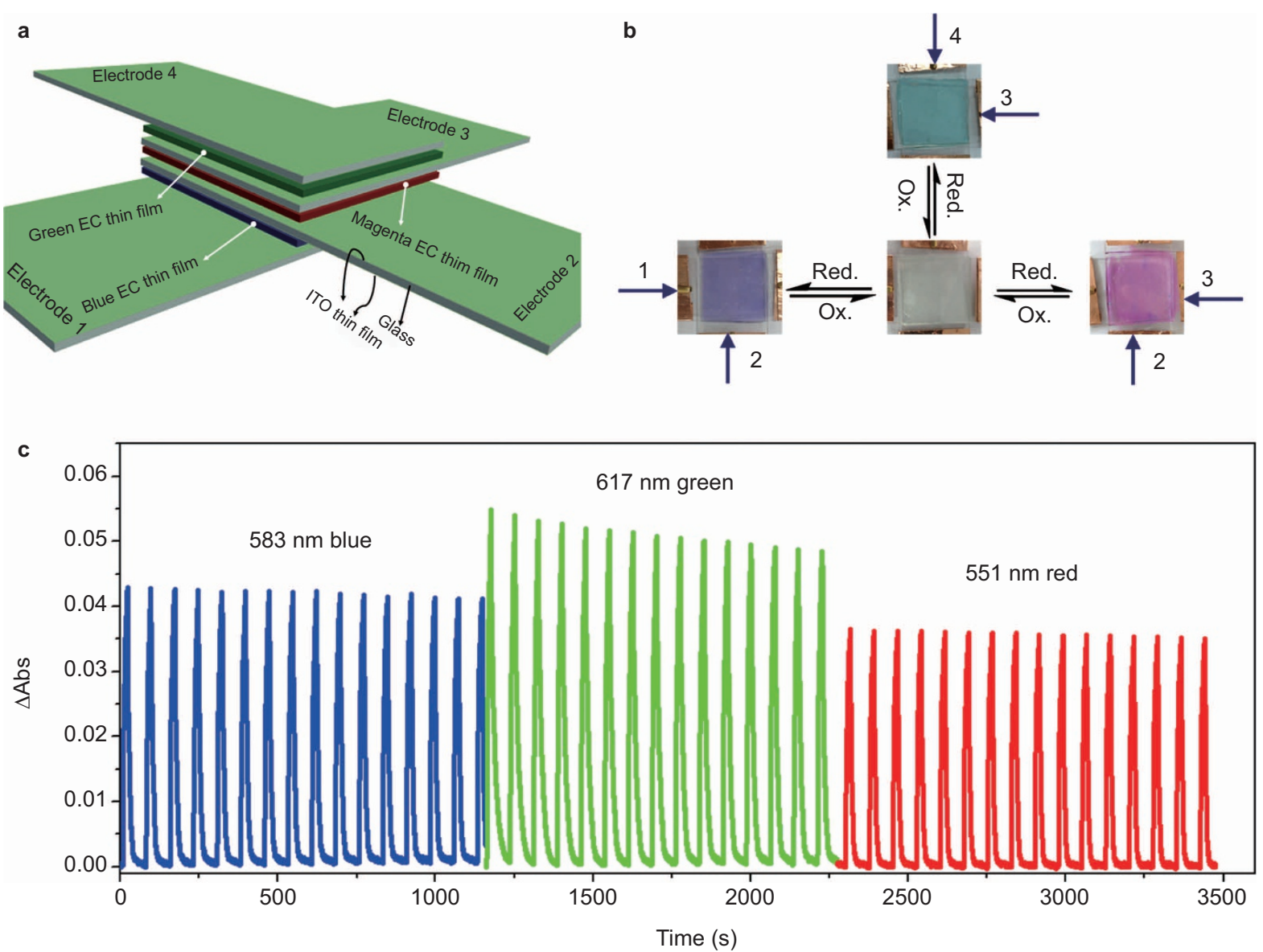

Figure 6 (a) Schematic of the multi-layered EC thin-film sandwich device using multi-electrodes. (b) The real color of the laminated electrochromic ITO device after an alternative bias voltage (-3.5/2.0 V) was applied to the relevant electrodes (green from electrodes 3 and 4; blue from electrodes 1 and 2; magenta from electrodes 2 and 3.) (c) Absorbance variation ( $583 \mathrm{~nm}$ blue, $617 \mathrm{~nm}$ green, $551 \mathrm{~nm}$ red) of laminated EC ITO device by alternating $-3.0 \mathrm{~V}$ and $2.0 \mathrm{~V}$ voltages. EC, electrochromic; ITO, indium tin oxide.

spectroscopy (Figure 6c), which indicated that this type of device could be very stable in an inert environment. To detect any blooming problems in our displays, which are usually associated with electrochromic devices due to the diffusion of the redox states, ${ }^{37,38}$ we carefully examined displayed words under a microscope; we were pleased to discover that the characters were very clear with good resolution, such as the 'JLU' shown in Supplementary Fig. S3.

Note that a multicolor display can be realized by either monolayered pixilated-device technology or the multi-layered stacked-electrodes strategy. For high-resolution multicolor displays, mono-layered pixilated devices are a better choice in practice; however, the manual construction is challenging in a lesser-equipped lab. We can employ a recently demonstrated high-resolution digital printing technology ${ }^{36,39}$ to complete the pixilated medium engineering tasks and related highresolution multicolor device. Regarding the multi-layered stacked-electrode strategy demonstrated here, its immediate applications may be suitable for medium- or low-resolution multicolor applications, such as a smart window or a large electronic billboard. For its potential application in a high-resolution display, the major challenge of the precise alignment of the multi-layers should be resolved to prevent problems in the processing of numerous layers of pixilated devices with high resolution during manufacture of the device. In addition, effective and superior lamination of semisolid electrolytes may also prove challenging, and crosslinking or insolubilization of the previous layers will be needed during the process. Although many technical challenges for the multi-layered device need to be resolved, we remain optimistic regarding its application potential because several excellent technologies have been developed to overcome these challenges, such as in situ photo-crosslinking ${ }^{40,41}$ and patterned Ag electrodes ${ }^{37,38}$ combined with slot die coating. We will take advantage of all existing technologies and explore how to make the process feasible for highresolution multi-layered devices.

\section{Understanding the electrochromic reaction mechanism}

Although this study has progressed with many satisfactory results, the exact mechanism of this electrochemical color switching needs to be clarified. To completely understand the mechanism, detailed CV experiments of TM1 and two reference compounds (TM2 and TM3) were conducted; their corresponding results $\left(E_{\mathrm{o}}\right)$ are summarized in Table 1.

Table 1 Reduction potentials of TM1, TM2 and TM3.

\begin{tabular}{llcc}
\hline & $E_{\text {red1 }}(\mathrm{V})$ & $E_{\text {red2 }}(\mathrm{V})$ & $E_{\text {red3 }}(\mathrm{V})$ \\
\hline TM1 & -1.22 & -1.7 & -2.0 \\
TM2 & -2.0 & - & - \\
TM3 & -1.20 & -1.92 & - \\
\hline
\end{tabular}


First, we investigated the redox behaviors of the molecules. The perspicuous reduction peak of TM2 at $-2.0 \mathrm{~V}$ can be assigned to the reduction of the carbonyl in ketone. The reduction peaks of TM3 with a nitro, which appeared at $-1.20 \mathrm{~V}$ and $-1.92 \mathrm{~V}$, can be assigned as the first reduction and the second reduction of its nitro. TM1 with both nitro and carbonyl groups produces a more complex cyclic voltammogram with three reduction peaks within the range of $-2.0 \mathrm{~V}$ to $+0.3 \mathrm{~V}$. The first reduction peak $(-1.22 \mathrm{~V})$ of TM1 can be affiliated with the first reduction of its nitro group by comparison with TM3 $(-1.2 \mathrm{~V})$. The assignment of the remaining two reduction peaks remains uncertain because the reduction potentials of the ketone group and the second reduction of the nitro group fall within a similar range. However, the second reduction peak of TM1 $(-1.7 \mathrm{~V})$ is tentatively inferred to its carbonyl reduction based on the known fact that the reduction potential of methyl ketone is inversely proportional to the acidity of $\alpha-\mathrm{H}$ in its adjacent methylene unit ${ }^{42}$ and the second reduction peak of the nitro group usually emerges at approximately $-2.0 \mathrm{~V}$ in these conditions. Thus, the third reduction peak of TM1 is correlated to the second reduction peak of the nitro group. This correlation is consistent with two nitro-containing methyl ketone derivatives in our previous study. ${ }^{26}$

Next, we investigated the structure of the electrically generated colored intermediate. From the previous investigation, we determined that the electrochromic absorption peak of TM1 occurs at $617 \mathrm{~nm}$ (by $-1.8 \mathrm{~V}$ or $-1.3 \mathrm{~V}$, Figure $4 \mathrm{~b}$ ), which coincides with the enolate isomer of TM1 (TM1-enolate) obtained from the treatment with chemical base (Figure $3 \mathrm{~b}$ ). This finding indicates that the electrically generated colored intermediate is a TM1-enolate.

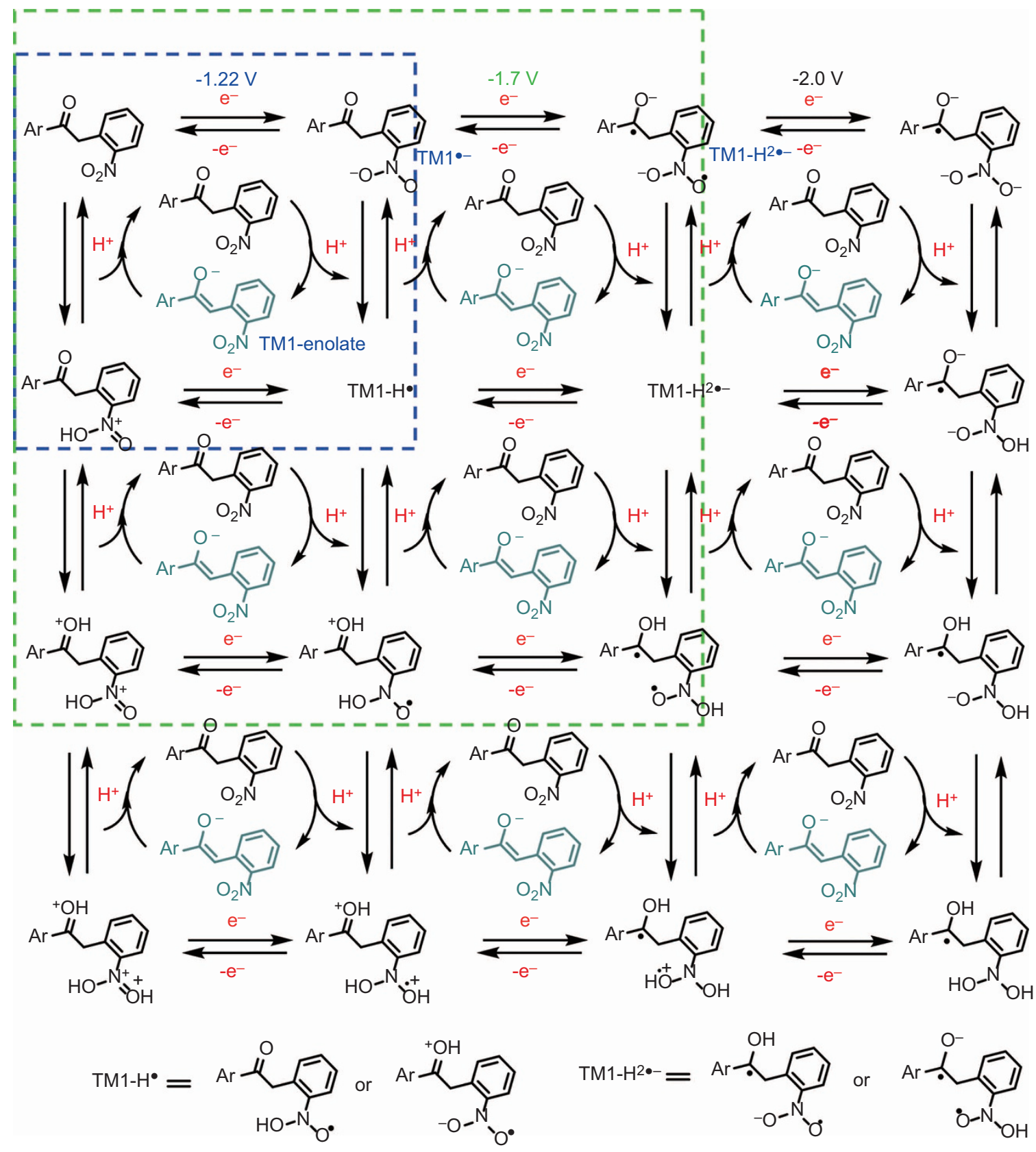

Figure 7 The electrochromic mechanism of TM1 in DMF involving triple-electron-coupled proton transfer (electrobase). DMF, dimethylformamide. 
Then, we sought to understand the electrochromic mechanism of the molecule. To understand the electrochromic process, detailed in situ measurements of the spectroelectrochemical behavior of TM1 were taken with a combined CV and ultraviolet-visible spectrometer. As shown in Figure 4a, the magnitude of the absorption peak at $617 \mathrm{~nm}$ begins to increase when the first reduction peak appears at $-1.0 \mathrm{~V}$, which is inferred to the first reduction of the nitro. This absorption increases after the second reduction peak appears at $-1.7 \mathrm{~V}$, which is inferred to the reduction of carbonyl. This absorption increases continuously after the third reduction peak appears at $-2.0 \mathrm{~V}$. This absorption peak continues to increase when the potential is switched until the potential is higher than the turn-on position of the first reduction peak; it decreases with time and then vanishes.

These data demonstrate that the electrochromism of TM1 is caused by radical anions from the reduction of both the carbonyl group and the nitro group and that the electrically generated colored intermediate is a TM1-enolate. These findings indicate that the corresponding TM1-enolate is most likely obtained through electron-coupled proton transfer, ${ }^{43,44}$ in which radical anions of nitro and carbonyl groups serve as the 'electro-base ${ }^{26,28}$ to extract the proton from the methylene unit of TM1 to induce its enolization.

We assessed whether the radical anion-induced proton transfer proceeds intermolecularly or intramolecularly. Both theoretical calculations and experimental evidence strongly suggest that the reaction occurs via a path of intermolecular proton transfer. Related density functional theory calculations show that the intermolecular proton transfer from the methyl ketone of TM1 to the nitro radical of TM1 is endothermic by $43.5 \mathrm{~kJ} \mathrm{~mol}^{-1}$ (Supplementary Fig. S4), which is significantly smaller than the intramolecular proton transfer $\left(69.8 \mathrm{~kJ} \mathrm{~mol}^{-1}\right)$. This result convinces us that intermolecular proton transfer is the reaction path because it is more favorable in thermodynamics. In addition, the experimental results from the spectroelectrochemical measurement of TM3 (Supplementary Fig. S5 and related discussion) show a distinct change in its absorption spectrum after its nitro group is reduced to nitro radical at $-1.4 \mathrm{~V}$. This result implies that if the reaction proceeds through intramolecular proton transfer, the absorption peak of the enolate product from the nitro radical of TM1 (TM1-RH-intra, Supplementary Fig. S5) should not be identical to the absorption peak of the TM1-enolate that was generated from a chemical base. However, the experimental observations (Figure 3b) contradict the analogy that is based on the hypothesis of the intramolecular proton transfer, which emphasizes that the electrochromic reaction of TM1 is an intermolecular proton transfer reaction.

Based on the results of the study, we suggest a possible mechanism for the electrochromic reaction of TM1. The entire electrochromic process presumably involves a triple-electron-coupled proton transfer, which is summarized in a potential-proton diagram ${ }^{45}$ (Figure 7). This diagram, which is commonly referred to as a Pourbaix diagram, expresses the relationship of protonated species and various redox. The horizontal line represents the electron transfer, the vertical line represents the proton transfer, and the insert cycle represents the reversible color change. At a lower potential of $-1.22 \mathrm{~V}$ versus SCE (saturated calomel electrode), TM1 is reduced by one electron to a fairly reactive $\mathrm{TM}^{\circ}{ }^{\circ-}$, which readily extracts a proton from an adjacent TM1 molecule to form a TM1- $\mathbf{H}^{\bullet}$ and a deprotonated TM1enolate (blue box in Figure 7). At a higher potential of $-1.7 \mathrm{~V}$ versus SCE, the reaction involving double-electron-coupled proton transfer was observed, as shown in the green box of Figure 7. A more reactive $\mathrm{TM}^{2 \cdot-}$ is produced by a two-electron reduction of TM1 and rapidly captures one or two protons from nearby TM1 molecules to generate corresponding TM1-enolate(s). When the bias potential is increased to $-2.0 \mathrm{~V}$ or higher versus SCE, the electrochromic process becomes more complex and will involve triple-electron-coupled proton transfer. The highly reactive intermediate that is generated via the tripleelectron reduction of the TM1 will promptly grab proton(s) from the encountered TM1 molecule(s) and generate one to three TM1-enolate(s). Thus, the higher the potential, the larger is the amount of TM1-enolate that will be generated and the faster is the electrochromic reaction.

\section{CONCLUSIONS}

The basochromism, solvatochromism and electrochromism of a novel methyl ketone bridged molecule TM1 have been investigated by CV, in situ ultraviolet-visible spectroscopy and density functional theory. By integrating the technologies of medium engineering, in situ electrobase and laminated electrodes, three primary colors (green, blue and magenta) from a single-molecule thin-film device have been demonstrated with reasonable control for creating multicolor. This electrochromic material is durable with a high coloration efficiency (350 $\left.\mathrm{cm}^{2} \mathrm{C}^{-1}\right)$, fast switching time $(50 \mathrm{~ms})$ and superior reversibility. In this study, a solvatochromic $\mathrm{pH}$-sensitive material has been applied in multicolor electrochromic materials by in situ 'electro-acid/base'. We believe that the concept of 'electro-acid/base' and tuning the materials' functionality via a media-engineering approach will not only open a new avenue for color-tuning, besides the structural design of the colorant, but also inspire further development in many other properties of tuning by this medium engineering approach, such as conductance and the redox property. Versatile applications in data recording, ultrathin flexible display and optical communication and sensing can thereby be realized.

\section{ACKNOWLEDGEMENTS}

This study was supported by the National Science Foundation of China (Grant No. 51373068) and program for Chang Jiang Scholars and Innovative Research Team in University (No. IRT101713018). The authors also acknowledge E.K. Wang, Y Fang and H Cheng for their assistance.

1 Mortimer RJ. Organic electrochromic materials. Electrochim Acta 1999; 44: 2971 2981.

2 Beaujuge PM, Reynolds JR. Color control in $\pi$-conjugated organic polymers for use in electrochromic devices. Chem Rev 2010; 110: 268-320.

3 Mortimer RJ, Dyer AL, Reynolds JR. Electrochromic organic and polymeric materials for display applications. Displays 2006; 27: 2-18.

4 Michaelis A, Berneth H, Haarer D, Kostromine S, Neigl R et al. Electrochromic dye system for smart window applications. Adv Mater 2001; 13: 1825-1828.

5 Kalagi SS, Mali SS, Dalavi DS, Inamdar AI, Im H et al. Limitations of dual and complementary inorganic-organic electrochromic device for smart window application and its colorimetric analysis. Synth Met 2011; 161: 1105-1112.

6 Andersson $\mathrm{P}$, Nilsson D, Svensson PO, Chen M, Malmström A et al. Active matrix displays based on all-organic electrochemical smart pixels printed on paper. $A d v$ Mater 2002; 14: 1460-1464.

7 Zirkl M, Sawatdee A, Helbig U, Krause M, Scheipl G et al. An all-printed ferroelectric active matrix sensor network based on only five functional materials forming a touchless control interface. Adv Mater 2011; 23: 2069-2074.

8 Andersson $\mathrm{P}$, Forchheimer $\mathrm{R}$, Tehrani $\mathrm{P}$, Berggren $\mathrm{M}$. Printable all-organic electrochromic active-matrix displays. Adv Funct Mater 2007; 17: 3074-3082.

9 Yagi M, Sone K, Yamada M, Umemiya S. Preparation and multicolor electrochromic performance of $\mathrm{a} \mathrm{WO}_{3}$ /tris-(2,2'-bipyridine)ruthenium(II)/polymer hybrid film. Chem Eur J 2005; 11: 767-775.

10 Song HK, Lee EJ, Oh SM. Electrochromism of 2,2'-azinobis(3-ethylbenzothiazoline6-sulfonate) incorporated into conducting polymer as a dopant. Chem Mater 2005; 17: 2232-2233.

11 Xia XH, Tu JP, Zhang J, Huang XH, Wang XL et al. Multicolor and fast electrochromism of nanoporous $\mathrm{NiO} /$ poly(3,4-ethylenedioxythiophene) composite thin film. Electrochem Commun 2009; 11: 702-705. 
12 Yen $\mathrm{HJ}$, Lin KL, Liou GS. Transmissive to black electrochromic aramids with high nearinfrared and multicolor electrochromism based on electroactive tetraphenylbenzidine units. J Mater Chem 2011; 21: 6230-6237.

13 Sharmoukh W, Ko KC, Ko JH, Jung IG, Noh C et al. Designed synthesis of ferrocenylanthraquinones and their bifunctional electrochromic properties. Org Lett 2010; 12: 3226-3229.

14 Sun J, Lv X, Wang P, Zhang Y, Dai Y et al. A donor-acceptor cruciform $\pi$-system: high contrast mechanochromic properties and multicolour electrochromic behavior. J Mater Chem C 2014; 2: 5365-5371.

15 Liu Y, Lagrost C, Costuas K, Tchouar N, Bozec HL et al. A multifunctional organometallic switch with carbon-rich ruthenium and diarylethene units. Chem Commun 2008: 6117-6119.

16 Puodziukynaite E, Oberst JL, Dyer AL, Reynolds JR, Establishing dual electrogenerated chemiluminescence and multicolor electrochromism in functional ionic transitionmetal complexes. J Am Chem Soc 2012; 134: 968-978.

17 Motiei L, Lahav M, Freeman D, van der Boom ME. Electrochromic behavior of a selfpropagating molecular-based assembly. J Am Chem Soc 2009; 131: 3468-3469.

18 Wen RT, Niklasson GA, Granqvist CG. Electrochromic iridium oxide films compatibility with propionic acid, potassium hydroxide, and lithium perchlorate in propylene carbonate. Sol Energy Mater Sol Cells 2014; 120: 151-156.

19 Abruna HD, Denisevich P, Umana M, Meyer TJ, Murray RW. Rectifying interfaces using two-layer films of electrochemically polymerized vinylpyridine and vinylbipyridine complexes of ruthenium and iron on electrodes. J Am Chem Soc 1981; 103: 1-5.

20 Liu W, Huang W, Pink M, Lee D. Layer-by-layer synthesis of metal-containing conducting polymers: caged metal centers for interlayer charge transport. J Am Chem Soc 2010; 132: 11844-11846.

21 Matsui J, Kikuchi R, Miyashita T. A trilayer film approach to multicolor electrochromism. J Am Chem Soc 2014; 136: 842-845.

22 Sharmoukh W, Ko KC, Noh C, Lee JY, Son SU. Designed synthesis of multielectrochromic systems bearing diaryl ketone and isophthalates. J Org Chem 2010 75: 6708-6711.

23 Chidichimo G, Imbardelli D, De Simone BC, Barone P, Barberio M et al. Spectroscopic and kinetic investigation of ethyl viologen reduction in novel electrochromic plastic films. J Phys Chem C 2010; 114: 16700-16705.

24 Yashiro T, Hirano S, Naijoh Y, Okada Y, Tsuji K et al. Novel design for color electrochromic display. SID Symp Dig Tech 2011; 42: 42-45.

25 Kobayashi N, Miura S, Nishimura M, Urano H. Organic electrochromism for a new color electronic paper. Sol Energy Mater Sol Cells 2008; 92: 136-139.

26 Zhang YM, Li M, Li W, Huang Z, Zhu S et al. A new class of "electro-acid/base"'induced reversible methyl ketone colour switches. J Mater Chem C 2013; 1: 53095314.

27 Zhang YM, Wang X, Li W, Zhang W, Li M et al. Bio-inspired enol-degradation for multipurpose oxygen sensing. Chem Commun 2014; 50: 13477-13480.

28 Zhang YM, Li W, Wang X, Yang B, Li M et al. Highly durable colour/emission switching of fluorescein in a thin film device using "electro-acid/base" as in situ stimuli. Chem Commun 2014; 50: 1420-1422.

29 Reichardt C. Solvatochromic dyes as solvent polarity indicators. Chem Rev 1994; 94 2319-2358.
30 Fletcher KA, Storey IA, Hendricks AE, Pandey S, Pandey S. Behavior of the solvatochromic probes Reichardt's dye, pyrene, dansylamide, Nile Red and 1pyrenecarbaldehyde within the room-temperature ionic liquid bmimPF 6 . Green Chem 2001; 3: 210-215.

31 Frisch MJ, Trucks GW, Schlegel HB, Scuseria GE, Robb MA et al. Gaussian 09 (Revision A. 02). Wallingford, CT: Gaussian, Inc.; 2009.

32 Zhao GJ, Yu F, Zhang MX, Northrop BH, Yang $\mathrm{H}$ et al. Substituent effects on the intramolecular charge transfer and fluorescence of bimetallic platinum complexes. J Phys Chem A 2011; 115: 6390-6393.

33 Bryce MR. Tetrathiafulvalenes as $\pi$-electron donors for intramolecular charge-transfer materials. Adv Mater 1999; 11: 11-23.

34 Jensen J, Hösel M, Kim I, Yu JS, Jo J et al. Fast switching ITO free electrochromic devices. Adv Funct Mater 2014; 24: 1228-1233.

35 Thakur VK, Ding G, Ma J, Lee PS, Lu X. Hybrid materials and polymer electrolytes for electrochromic device applications. Adv Mater 2012; 24: 4071-4096.

36 Sheng L, Li M, Zhu S, Li H, Xi G et al. Hydrochromic molecular switches for water-jet rewritable paper. Nat Commun 2014; 5: 3044.

37 Gupta R, Walia S, Hösel M, Jensen J, Angmo D et al. Solution processed large area fabrication of Ag patterns as electrodes for flexible heaters, electrochromics and organic solar cells. J Mater Chem A 2014; 2: 10930-10937.

38 Jensen J, Dam HF, Reynolds JR, Dyer AL, Krebs FC. Manufacture and demonstration of organic photovoltaic-powered electrochromic displays using roll coating methods and printable electrolytes. J Polym Sci Part B Polym Phys 2012; 50: 536-545.

39 Kuang M, Wang L, Song Y. Controllable printing droplets for high-resolution patterns. Adv Mater 2014; 26: 6950-6958.

40 Jensen J, Krebs FC. From the bottom up-flexible solid state electrochromic devices. Adv Mater 2014; 26: 7231-7234.

41 Jensen J, Dyer AL, Shen DE, Krebs FC, Reynolds JR. Direct photopatterning of electrochromic polymers. Adv Funct Mater 2013; 23: 3728-3737.

42 Kuhn A, von Eschwege KG, Conradie J. Electrochemical and density functional theory modeled reduction of enolized 1,3-diketones. Electrochim Acta 2011; 56: 62116218.

43 Kumar A, Sevilla MD. Proton-coupled electron transfer (PCET) in DNA on formation of radiation produced ion radicals. Chem Rev 2010; 110: 7002-7023.

44 Tan SL, Webster RD. Electrochemically induced chemically reversible proton-coupled electron transfer reactions of riboflavin (vitamin B2). J Am Chem Soc 2012; 134: 5954-5964.

45 Costentin C, Robert M, Savéant JM. Update 1 of: electrochemical approach to the mechanistic study of proton-coupled electron transfer. Chem Rev 2010; 110: PR1PR40.

\section{(c) (i) $(-)$ This work is licensed under a Creative Commons Attribution-}

(c) 1 BY NC ND NonCommercial-NoDerivs 3.0 Unported License. The images or other third party material in this article are included in the article's Creative Commons license, unless indicated otherwise in the credit line; if the material is not included under the Creative Commons license, users will need to obtain permission from the license holder to reproduce the material. To view a copy of this license, visit http://creativecommons.org/licenses/ by-nc-nd/3.0/ 